\title{
Scaling the Decay of Turbulence Kinetic Energy in the Free-Convective Boundary Layer
}

\author{
Omar El Guernaoui ${ }^{1}$ D Joachim Reuder ${ }^{1} \cdot$ Igor Esau $^{2} \cdot$ Tobias Wolf $^{2}$. \\ Björn Maronga ${ }^{3,4}$
}

Received: 15 October 2018 / Accepted: 8 May 2019 / Published online: 12 June 2019

(c) The Author(s) 2019

\begin{abstract}
We investigate the scaling for decaying turbulence kinetic energy (TKE) in the free-convective boundary layer, from the time the surface heat flux starts decaying, until a few hours after it has vanished. We conduct a set of large-eddy simulation experiments, consider various initial convective situations, and prescribe realistic decays of the surface heat flux over a wide range of time scales. We find that the TKE time evolution is dictated by the decaying magnitude of the surface heat flux up to $0.7 \tau$ approximately, where $\tau$ is the prescribed duration from maximum to zero surface heat flux. During the time period starting at zero surface heat flux, we search for potential power-law scaling by examining the log-log presentation of TKE as a function of time. First, we find that the description of the decay highly depends on whether the time origin is defined as the time when the surface heat flux starts decaying (traditional scaling framework), or the time when it vanishes (proposed new scaling framework). Second, when varying $\tau$, the results plotted in the traditional scaling framework indicate variations in the power-law decay rates over several orders of magnitude. In the new scaling framework, however, we find a unique decay exponent in the order of 1, independent of the initial convective condition, and independent of $\tau$, giving support for the proposed scaling framework.
\end{abstract}

Keywords Convective scaling · Free-convective boundary layer · Power-law scaling · Similarity relations - Turbulence kinetic energy decay

\section{Introduction}

Daytime atmospheric turbulent convection typically develops over heated surfaces, achieving the most intense mixing in the early afternoon, and decays following the subsequent reduction

$\triangle$ Omar El Guernaoui

omarelguernaoui@gmail.com

1 Geophysical Institute, University of Bergen, and Bjerknes Centre for Climate Research, Bergen, Norway

2 Nansen Environmental and Remote Sensing Centre, and Bjerknes Centre for Climate Research, Bergen, Norway

3 Institute of Meteorology and Climatology, Leibniz University Hannover, Hannover, Germany

4 Geophysical Institute, University of Bergen, Bergen, Norway 
in the surface sensible heat flux $(H)$ during the afternoon transition. The "surface sensible heat flux" will hereafter be called the "heat flux". The sketch in Fig. 1 illustrates the main stages of turbulence decay in the convective boundary layer (CBL); we define these stages after Nadeau et al. (2011). The first stage is an afternoon transition, and at this stage, the heat flux decreases but remains positive, thus, counteracting the turbulence decay. The second stage is an evening transition. At this stage, the heat flux becomes negative, and a shallow stably-stratified boundary layer (SBL) insulates the residual convective turbulence in the atmosphere from the surface.

Significant understanding of the afternoon and evening transitions in the CBL was achieved following the Boundary-Layer Late Afternoon and Sunset Turbulence (BLLAST) project (Lothon et al. 2014). The BLLAST project was primarily a field experiment, which integrated a complete hierarchy of modelling efforts (e.g. Darbieu et al. 2015; Couvreux et al. 2016; Nilsson et al. 2016). Our idealized study focuses on scaling the bulk turbulence kinetic energy (TKE) response to the heat-flux decay. We investigate the decay of TKE in the CBL, and how the corresponding decay laws relate to other configurations of decaying turbulence.

The first motivation is based on van Heerwaarden and Mellado (2016), who used a direct numerical simulation (DNS), and suggested that the decay of TKE in the CBL during the afternoon transition is a direct function of the time-dependent heat flux and the depth of the mixed layer $\left(z_{i}\right)$, namely the convective scaling established by Deardorff (1970). However, van Heerwaarden and Mellado (2016) provided little insight into the very late stage of the decay when the magnitude of the heat flux, although positive, becomes too small to scale the decay of TKE (see their Fig. 6d, green curve). With our set of large-eddy simulation (LES) experiments, we first show that our results are in line with van Heerwaarden and Mellado (2016). Taking a step further, we quantify the time when the convective scaling breaks down, and relate our result to Darbieu et al. (2015).

The second motivation comes from Nadeau et al. (2011) who suggested a simple local model for the decay of both the afternoon and evening transitions. Based on the TKE budget

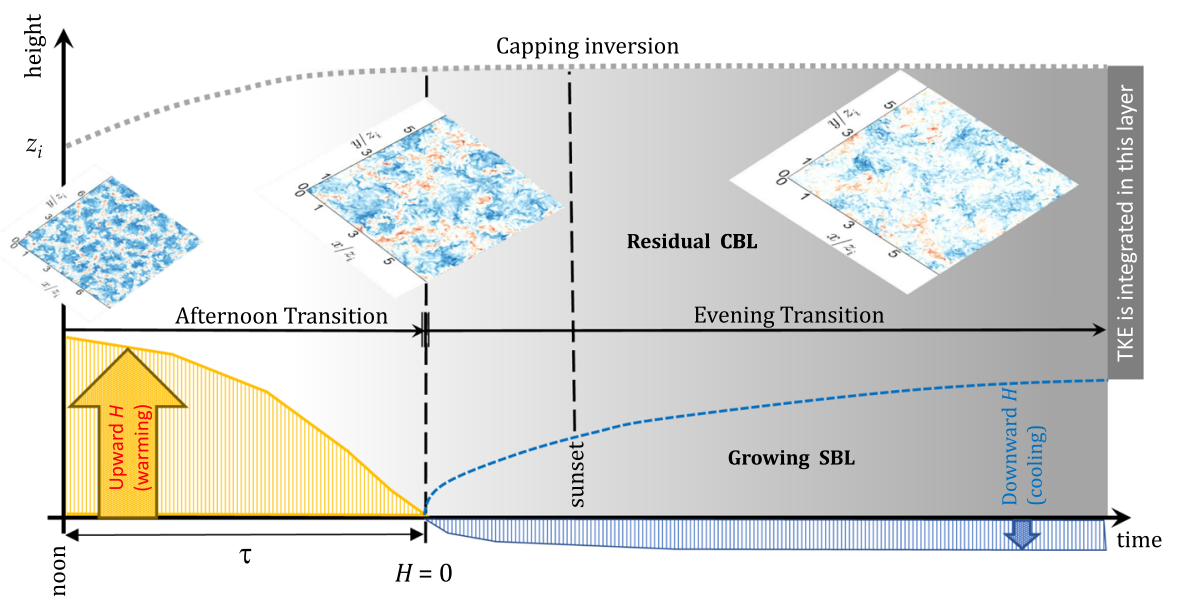

Fig. 1 Schematic sketch for the decay of turbulent convective mixing in the atmospheric boundary layer, responding to the decay of the heat flux. The decay phase is partitioned into afternoon and evening transitions, based on the time evolution of the heat flux. The height of the capping inversion is referred to by $z_{i}$. The horizontal cross-sections show corresponding typical instantaneous fields of vertical velocity. The graphs are taken from model simulations performed herein 
equation, they prescribed the buoyancy production/destruction term according to surface observations from different field campaigns, and parametrized the dissipation term assuming a constant dissipation length scale (see their Eqs. 3 and 9). They solved for TKE by numerical integration, then plotted the solution in a log-log presentation, searching for linear regions in the curve that would suggest a power-law decay. The major finding in the TKE log-log presentation was a continuous increase of the absolute values of the slopes, around the time when the heat flux changes sign. To summarize the interpretation of this result, Nadeau et al. (2011) wrote: "Note how the decay rate goes from $t^{-2}$ to $t^{-6}$, indicating that the convective decay of turbulence starts slowly. The influence of stable stratification causes a rapid collapse of turbulence at the early evening transition." Rizza et al. (2013) ran a realistic LES experiment to verify that the conclusions of Nadeau et al. (2011) for the lateafternoon/early-evening transition still apply to the TKE averaged over the boundary-layer depth. We first show that our results are in line with Nadeau et al. (2011) and Rizza et al. (2013), then prove that the decay exponent in the scaling framework suggested by Nadeau et al. (2011) depends on $\tau$. We suggest a new power-law scaling for the residual TKE above the surface layer, with the corresponding exponent independent of $\tau$.

We make a distinction between the decay phases where, (i) the heat flux is positive and the convective scaling applies, and (ii) the heat flux is zero with remaining turbulence from the previous convective regime. For convenience, the latter phase is termed the evening transition. However, we keep in mind that zero heat flux is a limiting case for more realistic evening transition situations with negative heat flux and the formation of a near-surface SBL. We will show in the discussion that the power-law scaling suggested for the first-order decay of TKE within the core of the residual layer is not affected by the absence of a near-surface SBL.

Power-law scaling for decaying TKE has strong theoretical foundations in homogeneous and isotropic turbulence (e.g. von Karman and Howarth 1937; Kolmogorov 1941; Batchelor and Townsend 1948; Saffman 1967; George 1992). Von Karman and Howarth (1937) started with the prognostic equation for the two-point velocity correlation, and showed that power-law scaling for the TKE follows from the self-similarity assumption. Though most of the theoretical results agree upon a power-law decay, they diverge with respect to the corresponding decay exponent, predicted to be in the interval [1,2.5]. This is explained by the various choices one can make for the length and velocity scales within the assumed selfsimilar profile, and the wide range of Reynolds-number regimes (e.g. Meldi and Sagaut 2013; Djenidi and Antonia 2015). Power-law decay is further supported by DNS (e.g. Huang and Leonard 1994; Mazzi and Vassilicos 2004; Ishida et al. 2009; Perot 2011), and laboratory experiments (e.g. Batchelor and Townsend 1948; Monin and Yaglom 1975; Mohamed and LaRue 1990; Lavoie et al. 2007). Except in theory, where exact values for the decay exponent can be deduced, all numerical and laboratory experiments (to our knowledge) rely on fitting their results with analytical functions. We refer to Perot (2011, Sect. B) for a clear and critical appraisal of the issues raised by curve fitting.

In homogeneous and isotropic turbulence, the fields of velocity disturbances and associated TKE are-by definition - free to decay, independent of any forcing. Conversely, in the CBL, the decay of TKE during the afternoon transition is subject to the remaining thermal forcing at the surface. Even if one can assume a decoupling between the thermal sink at the surface, and the TKE evolution above the stable surface layer, decaying entrainment at the top inversion (e.g. Sorbjan 1997; Pino et al. 2006) might influence the TKE budget above the surface layer, through a conversion between kinetic, and potential energy.

Increased complexity in the CBL turbulence compared to homogeneous and isotropic turbulence, prevents the development of strong theories. Except in the simple model of Nieuwstadt and Brost (1986), based on the TKE budget without buoyancy leading to a 
power-law decay with exponent 2, almost all the conclusions for the turbulence decay rates in the CBL rely on fitting numerical results (e.g. Nieuwstadt and Brost 1986; Sorbjan 1997; Goulart et al. 2003; Pino et al. 2006; Nadeau et al. 2011; Rizza et al. 2013). With a new set of LES experiments, we examine the decay during the evening transition based on the $\log -\log$ presentation of TKE. In this presentation, we carefully analyze the consequences of two different choices for the time origin, and thereby question the currently accepted powerlaw scaling suggesting a fast turbulence decay during the evening transition. Based on this analysis, we then propose a new scaling that is given a physically sound basis when varying the physical parameters defining the system. Section 2 presents the LES model and describes the numerical experiments. Results are presented in Sect. 3, followed by a discussion in Sect. 4, and conclusions in Sect. 5.

\section{Description of the LES Model and the Numerical Experiments}

The LES model PALM (Maronga et al. 2015) (revision 2663) has been widely applied to different flow regimes in the convective and neutral boundary layers (e.g. Raasch and Franke 2011; Maronga 2014; Kanani-Sühring and Raasch 2015). The PALM model, which is used herein, solves the filtered Navier-Stokes equations in the Boussinesq approximation and the filtered transport equation for potential temperature. The model equations are discretized on a Cartesian grid using finite differences. A 1.5-order flux-gradient subgrid closure scheme after Deardorff (1980) is used, which involves the solution of an additional prognostic equation for the subgrid-scale TKE. A fifth-order advection scheme after Wicker and Skamarock (2002) and a third-order Runge-Kutta timestep scheme (Williamson 1980) are used to discretize the model in space and time, with all simulations in the present study carried out using cyclic lateral boundary conditions. For more details and a technical documentation, see Maronga et al. (2015).

Overall, we performed 38 LES runs in which the CBL is driven by a prescribed homogeneous heat flux. At the bottom boundary, we impose a non-slip condition, while at the top boundary within the free atmosphere, we impose constant velocity and potential temperature gradients. A damping layer is added to the highest levels of the modelling domain, to prevent effects of reflection within the boundary layer due to gravity waves formed at the interface between the boundary layer and the free atmosphere. The domain extends horizontally over $12.8 \mathrm{~km}$ in both $x$ and $y$ directions. In the vertical direction, the domain height is either set to $4.825 \mathrm{~km}$ for an initial kinematic surface heat flux of $0.1 \mathrm{~K} \mathrm{~m} \mathrm{~s}^{-1}$ or to $6.425 \mathrm{~km}$ for a heat flux of 0.2 or $0.3 \mathrm{~K} \mathrm{~m} \mathrm{~s}^{-1}$. These choices were guided by the requirement that the domain size should be at least two times (for the vertical) and five times (for the horizontal) larger than the maximum boundary-layer depth. This is sufficient to allow disturbances to develop independent of the periodic sidewall effects so that the largest turbulent eddies can evolve freely (Schmidt and Schumann 1989; de Roode et al. 2004). The grid spacing is $25 \mathrm{~m}$ in all three directions. With the boundary-layer height ranging from about 1000 to $2000 \mathrm{~m}$, our discretization is coarse compared to state-of-the-art, high resolution LES experiments (e.g. Sullivan and Patton 2011). However, a sensitivity test down to $12.5 \mathrm{~m}$ did not affect the results, since we focus on the first-order decay of the bulk TKE. In addition, the subgridscale TKE never exceeds $6 \%$ of the total TKE (except the first grid cells near the surface) through the time analysis for all the runs (see Fig. 2a for run $H 0.1 \_\tau 6 \_\lambda 3$ as an example [the nomenclature of the performed runs will be introduced later]). 
(a)

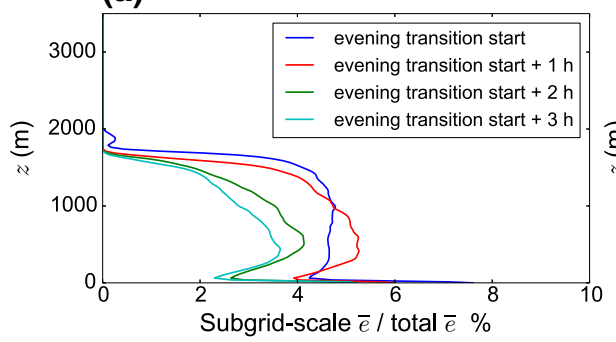

(b)

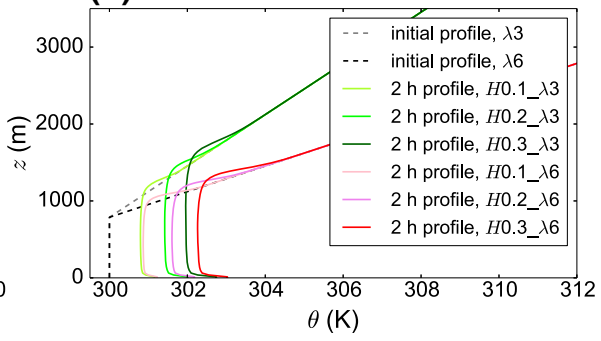

(c)

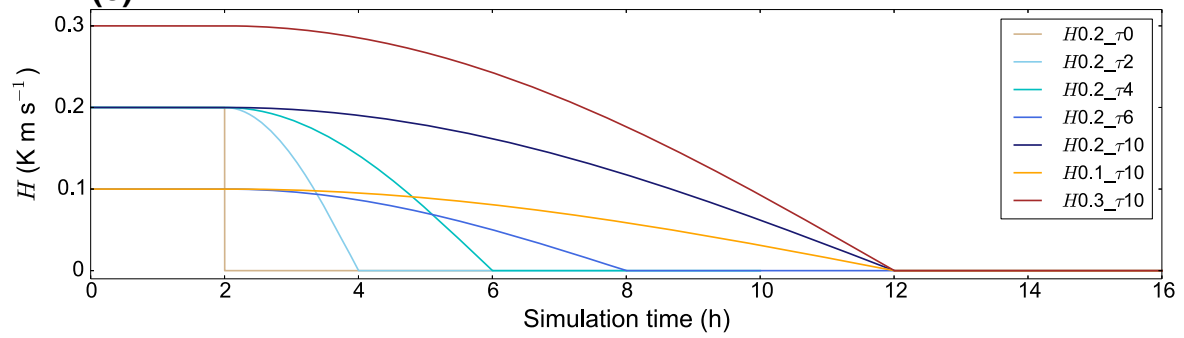

Fig. 2 a One-minute average profiles for the ratio of subgrid-scale and total TKE $(\bar{e})$, for run $H 0.1 \_\tau 6 \_\lambda 3$. b One-minute average potential temperature $(\theta)$ profiles at the simulation start (dashed lines), and at the afternoon transition start (solid lines). $\lambda$ is the free-atmospheric lapse rate in $\mathrm{K} \mathrm{km}^{-1}$. c Prescribed shapes for $H$

We focus on simulations with zero geostrophic wind speed in order to avoid further complexity of the flow. However, the effect of geostrophic background flow is briefly discussed in Sect. 4.2. All simulations are initiated with one of the two potential temperature profiles (dashed lines) shown in Fig. 2b. The potential temperature equals $300 \mathrm{~K}$ at the surface, and is constant up to $800 \mathrm{~m}$, while above it increases at either 3 or $6 \mathrm{~K} \mathrm{~km}^{-1}$. For each of these initial profiles, we use three values of the initial kinematic surface heat flux, $0.1,0.2$, and $0.3 \mathrm{~K} \mathrm{~m} \mathrm{~s}^{-1}$, resulting in six different convective situations. The convective flow develops during $2 \mathrm{~h}$ spin-up time, with constant heat flux. The initiation of the heat-flux decay marks the start of the afternoon transition, which is characterized by one of the six potential temperature profiles (solid lines) shown in Fig. 2b. In the remainder of this study, the initial conditions for the decay will refer to one of the six convective situations at the start of the afternoon transition.

To gain more confidence in our suggested scaling for the decay of TKE during the evening transition, we consider the durations $(\tau)$ of the afternoon transition, varying between 3 and $8 \mathrm{~h}$, based on ten intensive observation periods of the BLLAST field campaign (Lothon et al. 2014). For our simulations we selected $\tau=2,4,6$ and $10 \mathrm{~h}$, and prescribe a gradual and slow heat-flux decay with sinusoidal time dependence. The time between the maximum and zero heat flux corresponds to one quarter of the sinusoid, as shown in Fig. 2c. We considered additional runs with linear decay of the heat flux to test the sensitivity of our results to the prescribed shape of the heat flux. The evening transition starts when the heat flux reaches zero, with $H=0$ during $4 \mathrm{~h}$. We made the choice to investigate the decay of TKE when the surface thermal forcing is identically zero. This is of course a highly idealized situation, excluding complications rising from thermal-energy exchanges between the atmosphere and the surface. We debate the possible drawbacks from this simplifying assumption in Sect. 4. 
When discussing the relevance of the new scaling for the decay of TKE following a gradual decrease in the heat flux, it is also useful to consider a case where the heat flux abruptly decays to zero, as this situation has been investigated and discussed in various studies before (Nieuwstadt and Brost 1986; Pino et al. 2006).

Hereafter, we refer to each experiment by the initial value of the kinematic surface heat flux, followed by the duration of the afternoon transition, and finally the free atmospheric lapse rate. For example, in run $H 0.1 \_\tau 6 \_\lambda 3$ the initial kinematic surface heat flux is $0.1 \mathrm{~K} \mathrm{~m} \mathrm{~s}^{-1}$, and decreases to zero during the following $6 \mathrm{~h}$; the free atmospheric lapse rate is $\lambda=$ $3 \mathrm{~K} \mathrm{~km}^{-1}$. The runs with linear heat-flux decay are used only for comparison with the sinusoidal-decay cases. We therefore do not indicate the full nomenclature for these runs. Table 1 summarizes the convective scales at the start of the afternoon transition for the LES runs with zero geostrophic wind speed, along with an eddy-turnover time defined at the beginning of the evening transition, which will be introduced as a normalization factor in Sect. 3.4.2.

Table 1 Convective scales (after Deardorff (1970)) at the afternoon-transition start: $z_{i 0}, w_{* 0}$, and $t_{* 0}$ The subscript 0 indicates the afternoon transition start. The eddy-turnover time $t_{\text {ET }}$ is defined at the eveningtransition start as: $\left(z_{i} /[\bar{e}]^{1 / 2}\right)_{\mathrm{ET}}$. The square brackets indicate averaging over $z_{i}\left(z_{i}\right.$ is defined in Sect. 3.1)

\begin{tabular}{lllll}
\hline Run & $z_{i 0}(\mathrm{~m})$ & $w_{* 0}\left(\mathrm{~m} \mathrm{~s}^{-1}\right)$ & $t_{* 0}(\mathrm{~min})$ & $t_{\text {ET }}(\mathrm{min})$ \\
\hline$H 0.1 \_\tau 10 \_\lambda 3$ & 1275 & 1.6 & 13.2 & 68 \\
$H 0.1 \_\tau 6 \_\lambda 3$ & 1275 & 1.6 & 13.2 & 56 \\
$H 0.1 \_\tau 4 \_\lambda 3$ & 1275 & 1.6 & 13.2 & 50 \\
$H 0.1 \_\tau 2 \_\lambda 3$ & 1275 & 1.6 & 13.2 & 42 \\
$H 0.2 \_\tau 10 \_\lambda 3$ & 1550 & 2.2 & 12.0 & 66 \\
$H 0.2 \_\tau 6 \_\lambda 3$ & 1550 & 2.2 & 12.0 & 52 \\
$H 0.2 \_\tau 4 \_\lambda 3$ & 1550 & 2.2 & 12.0 & 47 \\
$H 0.2 \_\tau 2 \_\lambda 3$ & 1550 & 2.2 & 12.0 & 39 \\
$H 0.3 \_\tau 10 \_\lambda 3$ & 1775 & 2.6 & 11.4 & 65 \\
$H 0.3 \_\tau 6 \_\lambda 3$ & 1775 & 2.6 & 11.4 & 54 \\
$H 0.3 \_\tau 4 \_\lambda 3$ & 1775 & 2.6 & 11.4 & 42 \\
$H 0.3 \_\tau 2 \_\lambda 3$ & 1775 & 2.6 & 11.4 & 35 \\
$H 0.1 \_\tau 10 \_\lambda 6$ & 1125 & 1.5 & 12.2 & 61 \\
$H 0.1 \_\tau 6 \_\lambda 6$ & 1125 & 1.5 & 12.2 & 51 \\
$H 0.1 \_\tau 4 \_\lambda 6$ & 1125 & 1.5 & 12.2 & 45 \\
$H 0.1 \_\tau 2 \_\lambda 6$ & 1125 & 1.5 & 12.2 & 37 \\
$H 0.2 \_\tau 10 \_\lambda 6$ & 1275 & 2.0 & 10.5 & 55 \\
$H 0.2 \_\tau 6 \_\lambda 6$ & 1275 & 2.0 & 10.5 & 48 \\
$H 0.2 \_\tau 4 \_\lambda 6$ & 1275 & 2.0 & 10.5 & 45 \\
$H 0.2 \_\tau 2 \_\lambda 6$ & 1275 & 2.0 & 10.5 & 38 \\
$H 0.3 \_\tau 10 \_\lambda 6$ & 1425 & 2.4 & 9.9 & 54 \\
$H 0.3 \_\tau 6 \_\lambda 6$ & 1425 & 2.4 & 9.9 & 48 \\
$H 0.3 \_\tau 4 \_\lambda 6$ & 1425 & 2.4 & 9.9 & \\
$H 0.3 \_\tau 2 \_\lambda 6$ & 1425 & 2.4 & & \\
\hline & & & 9.9 & \\
\hline
\end{tabular}




\section{Results}

\subsection{Features of the Decay}

The analysis of turbulence decay carried out is based on the TKE averaged over the timeevolving CBL depth, which is defined as the height from the surface, where the vertical gradient of the mean potential temperature has its maximum. To give an impression of how the turbulent structures decay, Fig. 3 shows horizontal cross-sections for the vertical velocity $(w)$ at three different times for run $H 0.1 \_\tau 6 \_\lambda 3$. Each section is a snapshot at the level of $0.3 z_{i}$. At the start of the afternoon transition (Fig. 3a), our simulation reproduces the typical cellular organization in the flow structure for the stationary CBL with narrow updraft regions, surrounded by broad areas of downdraft. This is the classical expected flow field for a well-developed CBL (e.g. Schmidt and Schumann 1989). At the start of the evening transition (Fig. 3b), the gradients decreased and the disparity between updraft and downdraft areas is reduced. This is further quantified in Fig. 4, showing vertical profiles of the variance and skewness of $w$. The observed increase in horizontal length scales from Fig. 3a to $\mathrm{b}$ is consistent with the previous findings of Pino et al. (2006) and Darbieu et al. (2015). Around $9 t_{* 0}$ after the start of the evening transition (where the subscript 0 refers to the start of the afternoon transition, Fig. 3c) the structural distinction between updraft and downdraft areas has largely disappeared, consistent with the skewness approaching zero, indicating that updrafts and downdrafts are evenly distributed (Fig. 4b).

(a)

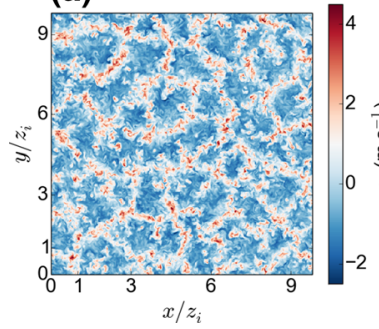

(b)

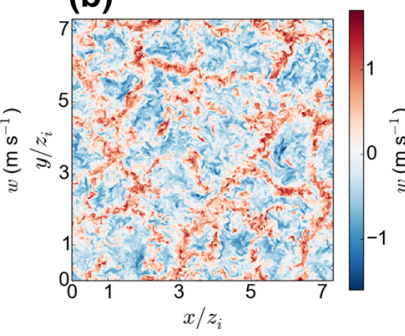

(c)

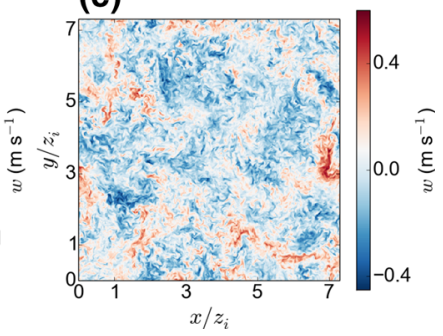

Fig. 3 Horizontal cross-sections of $w$ in run $H 0.1 \_\tau 6 \_\lambda 3$ at three times, a start of the afternoon transition; $\mathbf{b}$ start of the evening transition; $\mathbf{c} 9 t_{* 0}$ after the evening transition start. Each cross section is at $0.3 z_{i}$. Different colour scales are used for different times
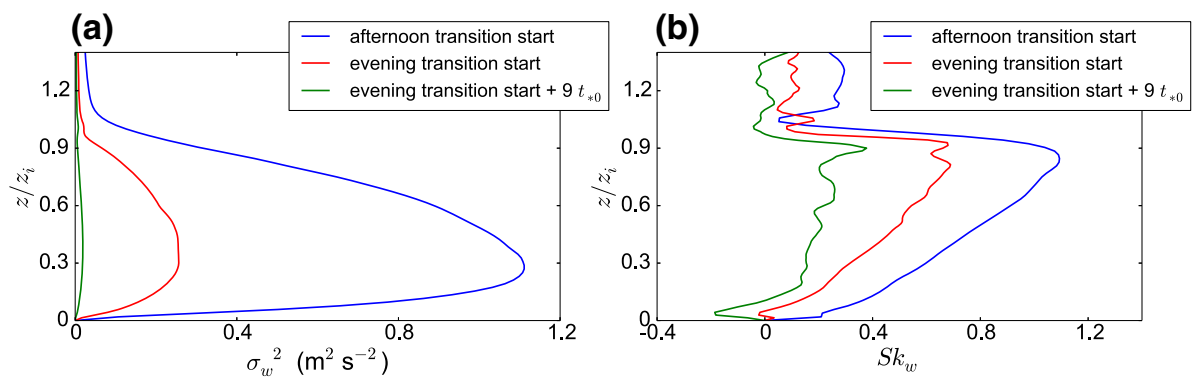

Fig. 4 Instantaneous profiles of the $w$ variance $\left(\sigma_{w}^{2}\right)(\mathbf{a})$ and skewness $\left(S k_{w}\right)(\mathbf{b})$, at the same time as shown for the horizontal cross-sections in Fig. 3 


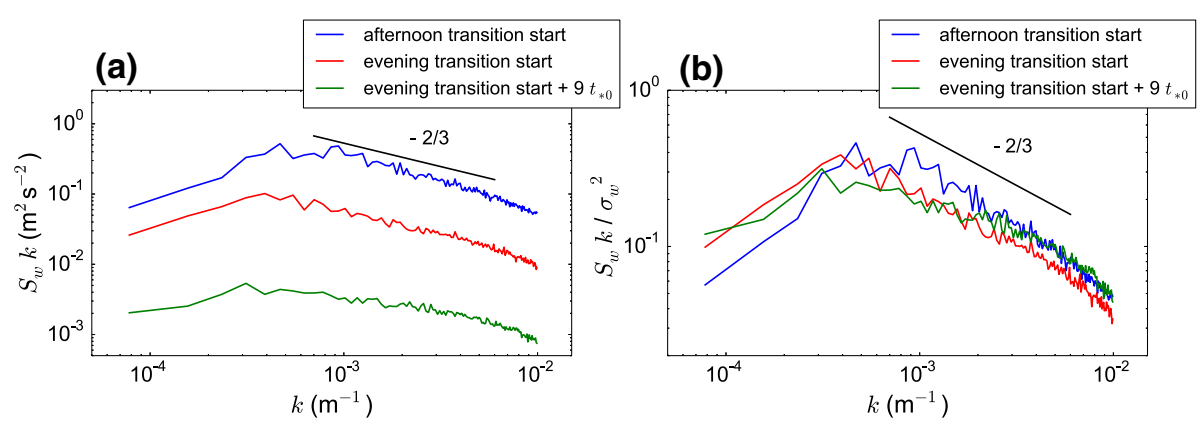

Fig. 5 Instantaneous $w$ spectra calculated along the $x$ direction averaged over the $y$ direction, at the same time as shown for the horizontal cross-sections in Fig. 3; a dimensional, b normalized by the variance. Contributions from wavelengths smaller than four grid spacing are not shown as these scales are unresolved by the LES model. The slope of the black lines is $-2 / 3$

One-dimensional spectra of the vertical velocity for three selected times are shown in Fig. 5. The spectral density decays at all scales (see Fig. 5a), and to further compare the relative qualitative decay among different scales, we normalize the spectral density with the variance of the respective data, and show the result in Fig. 5b. The peak of energy flattens and broadens through time, in line with the findings of Darbieu et al. (2015). In the inertial subrange, the time evolution of spectral density is not uniform across the scales, the largest scales being the most affected by the decay.

\subsection{The Break Down of Convective Scaling}

The convective scaling proposed by Deardorff (1970) applies when the heat flux is constant. In the case of decaying heat flux, van Heerwaarden and Mellado (2016) showed that the time dependent $w_{*}$ is still an appropriate scale for the decay of TKE. Furthermore, this result is subject to slowly varying heat flux (van Driel and Jonker 2011; van Heerwaarden and Mellado 2016). Using LES experiments, van Driel and Jonker (2011) showed that $w_{*}$ is no longer applicable when the heat flux varies rapidly, i.e. over time scales comparable to the initial convective eddy-turnover time. They suggested a new scaling factor which includes a time delay needed for the surface changes to be felt higher up in the atmosphere. Our results are in line with previous findings (see Fig. 6a, b), and show in addition a gradual transition around $0.8 \tau$, from the early to the late-afternoon transition where the time dependent $w_{*}$ becomes inapplicable as a scaling factor. This finding might be connected with the results of Darbieu et al. (2015). They suggested a separation of the afternoon transition in two phases: from 0 to $0.75 \tau$ when turbulence characteristics remain similar to those during fully convective conditions, and from 0.75 to $1 \tau$ when turbulence characteristics change rapidly. Prescribing a linear instead of sinusoidal decay of the heat flux does not change our conclusion, as can be seen in Fig. 6a, b.

As typical criteria used to define the CBL depth evolve differently during the afternoon transition (Lothon et al. 2014), we question the sensitivity of the transition from early to late afternoon, to the definition of the CBL depth. When we define the CBL depth as the height at which the heat flux is minimum instead of the height of the capping inversion, we find no change in the approximate time when the convective scaling breaks down (see Fig. 6b). During the late-afternoon transition and the following evening transition, the criteria based on the minimum heat flux cannot be applied to locate the top of the remnant layer of turbulence. 

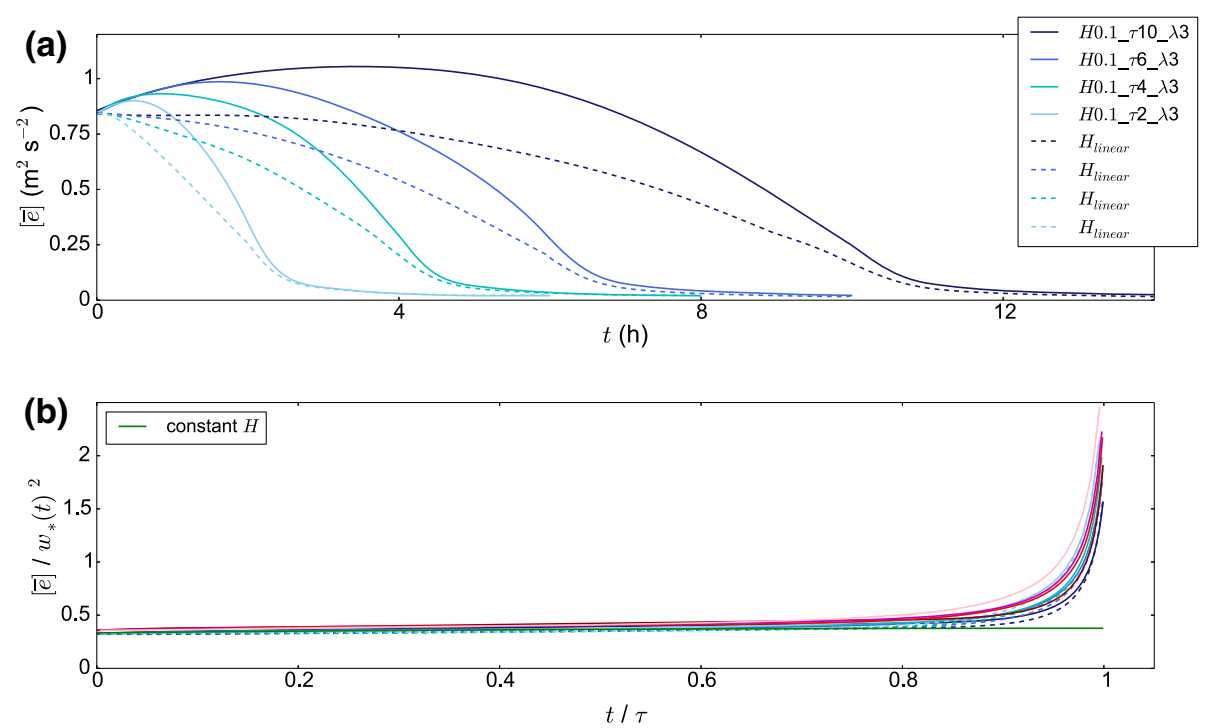

Fig. 6 Decay of TKE in runs $H 0.1 \_\tau 10 \_\lambda 3, H 0.1 \_\tau 6 \_\lambda 3, H 0.1 \_\tau 4 \_\lambda 3, H 0.1 \_\tau 2 \_\lambda 3$, and four additional runs with linear decay of $H$. a Dimensional decay; b Dimensionless decay. The time dependent $z_{i}$ (included in the definition of $w_{*}$ ) was taken as the height of the inversion for the blue curves, and the height of minimum heat flux for the red curves. A run with constant $H$ is also shown

We will use the criteria based on the capping inversion, which remains well defined even when the heat flux becomes zero. We cannot explore criteria based on the profiles of humidity or other tracers, because our study does not include these scalars.

\subsection{Interpretation of the TKE Decay in the Log-Log Presentation is Dependent on the Choice of the Time Origin}

As stated in the introduction, the time evolution of the TKE decay in the CBL is typically presented in a log-log plot. In Fig. 7a, the curve represents the time series of TKE $(\bar{e})$, averaged over the boundary layer depth, for run H0.1_ $\tau 6 \_\lambda 3$. Following Sorbjan (1997), Nadeau et al. (2011) and Rizza et al. (2013), we chose the time origin as the start of the afternoon transition, and the normalization factors for time and energy as the initial convective eddy-turnover time $t_{* 0}$ and the initial vertical-velocity scale $w_{* 0}{ }^{2}$. Hereafter, we refer to these scaling choices as the traditional scaling framework. In this framework, the qualitative shape of $[\bar{e}]$ is consistent with previous results (Sorbjan 1997; Nadeau et al. 2011; Rizza et al. 2013). In particular, during the late-afternoon transition, the absolute value of the slopes continuously increases in time.

Searching for potential power-law regimes in the traditional scaling framework during the evening transition, we plot in Fig. 7b a close-up view over this period of time. We observe two approximately linear regions, indicating two regions for potential power-law scaling for the decay of TKE: a first fast decay with an exponent of 9 , followed by a slower decay with an exponent of 3.4. We intentionally did not use any optimization method in order to select the time limit between the two approximate power laws, and calculate the linear fits. Therefore, attention should be drawn to the exponents' order of magnitude, not to the exact numerical values as specified in Fig. 7: a first fast $\left(\sim t^{-9}\right)$ decay during approximately $5 t_{* 0}$, and a 
(a)

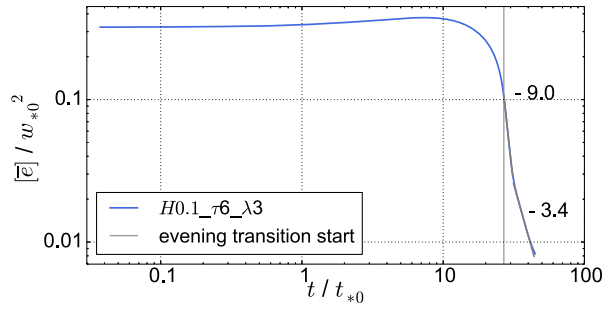

(c)

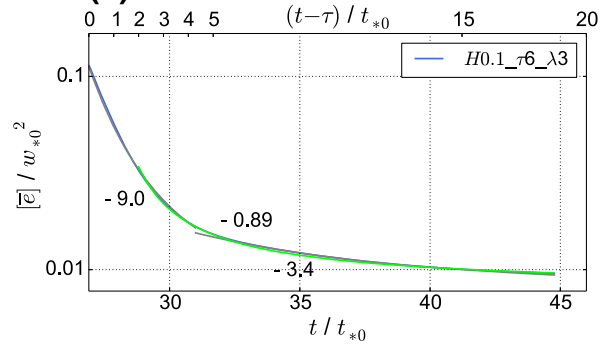

(b)

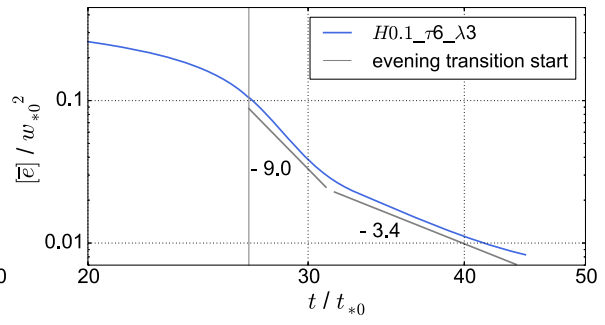

(d)

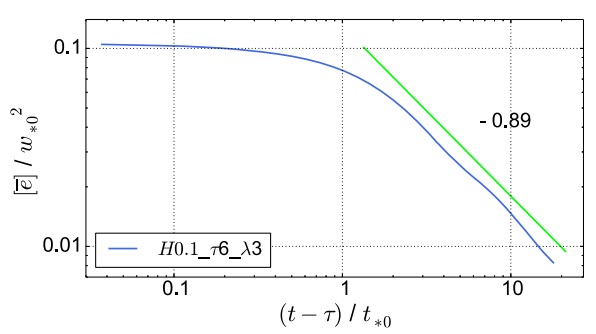

Fig. 7 Decay of TKE in run $H 0.1 \_\tau 6 \_\lambda 3$. a Log-log presentation where the time origin is defined as the afternoon transition start. The vertical line at 27.2 normalized time marks the beginning of the evening transition. b Close-up view over the evening transition. c Linear presentation. The normalized time variable is indicated on the lower axis and the upper axis, when the time origin is defined as the afternoon and evening transitions start respectively. The grey curves correspond to the power laws deduced from the log-log presentation in (b), the green curve corresponds to the power law deduced from the log-log presentation in (d), d log-log presentation where the time origin is defined as the evening transition start

second slower $\left(\sim t^{-3.5}\right)$ decay during approximately $13 t_{* 0}$. On the linear plot of Fig. $7 \mathrm{c}$ one sees that two power-law regimes, deduced from the log-log presentation on Fig. 7b, describe well the decay of TKE during the evening transition for run $H 0.1 \_\tau 6 \_\lambda 3$.

To introduce the proposed scaling framework, we consider a transformation for the time variable: $t \mapsto t-\tau \equiv \tilde{t}$, and plot $[\bar{e}]$ against $\tilde{t}$, in a $\log -\log$ presentation in Fig. $7 \mathrm{~d}$. The normalized time at the start of the evening transition is 0 . The time and energy units for normalization are still defined as the convective eddy-turnover time and the verticalvelocity scale at the start of the afternoon transition $\left(t_{* 0}\right.$ and $\left.w_{* 0}{ }^{2}\right)$. Around $2 t_{* 0}$ after the evening transition starts, a unique power law describes well the decay until the end of the simulation, and the corresponding decay exponent resulting from our fit is in the order of $1(n=0.87)$. On the linear plot of Fig. 7c showing the new power law (green curve), one sees that a unique power-law regime describes well the decay of TKE during the evening transition (after $\approx 2 t_{0}^{*}$ ). Note that in the linear presentation of $[\bar{e}]$, the shape of the curve is invariant to the time coordinate translation. We ran additional simulations (not shown) with prescribed linear decay of the heat flux; the results do not show deviations from the above conclusions.

For the log-log presentation of the TKE decay we observe that a simple transformation of the time coordinate leads to two different potential power-law scalings (Fig. 7b-d). For run $H 0.1 \_\tau 6 \_\lambda 3$, in the case where the time origin is defined as the start of the afternoon transition, two power laws are required to describe the decay $\left(\sim t^{-9}\right.$ and $\left.\sim t^{-3.5}\right)$. When the time origin is defined as the start of the evening transition, a single power law $\left(\sim t^{-1}\right)$ is sufficient for characterize the decay, after a period of time in the order of $t_{* 0}$. In the traditional 
scaling framework, the transition from the first to the second power law would indicate a physical transition from two distinct regimes. Such transition, however, has no physical basis in the absence of forcings and changes in the boundary conditions of the system. This physical argument is in favour of our suggested scaling, where a single power law applies. In the next section, we will give further support to the proposed scaling framework, by considering the response of the TKE decay to various initial convective situations at the start of the afternoon transition, and various durations of the afternoon transition.

One should be careful when interpreting the log-log presentation of $[\bar{e}]$ in Fig. 7d. During the first $t_{* 0}$ of the evening transition, the curve in Fig. $7 \mathrm{~d}$ gives the incorrect impression of a very small TKE decay rate. After this early stage, the absolute value of the decay rate seems to continuously increase in time, beyond approximately $2 t_{* 0}$, until the power law regime is reached. This qualitative behavior is not consistent with the linear presentation in Fig. 7c, where the decay of TKE is very fast during the first $t_{0}^{*}$ of the early-evening transition. The apparent very slow decay during the early-evening transition on the $\log -\log$ presentation of $[\bar{e}]$ is just a consequence of the stretching/squeezing of the time scale.

\subsection{Sensitivity of the Proposed Scaling Framework}

\subsubsection{Sensitivity to the Afternoon-Transition Duration and the Convective Initial Condition}

Four runs $\left(H 0.1 \_\tau 2 \_\lambda 3, H 0.1 \_\tau 4 \_\lambda 3, H 0.1 \_\tau 6 \_\lambda 3\right.$, and $\left.H 0.1 \_\tau 10 \_\lambda 3\right)$ help us to understand the sensitivity of the traditional and proposed scaling frameworks to the duration of the afternoon transition. Those runs have identical initial potential temperature and surface fluxes, but differ with respect to their prescribed transition time scale. Since $\tau$ varies, the normalized starting time of the evening transition also varies in the traditional scaling frame-
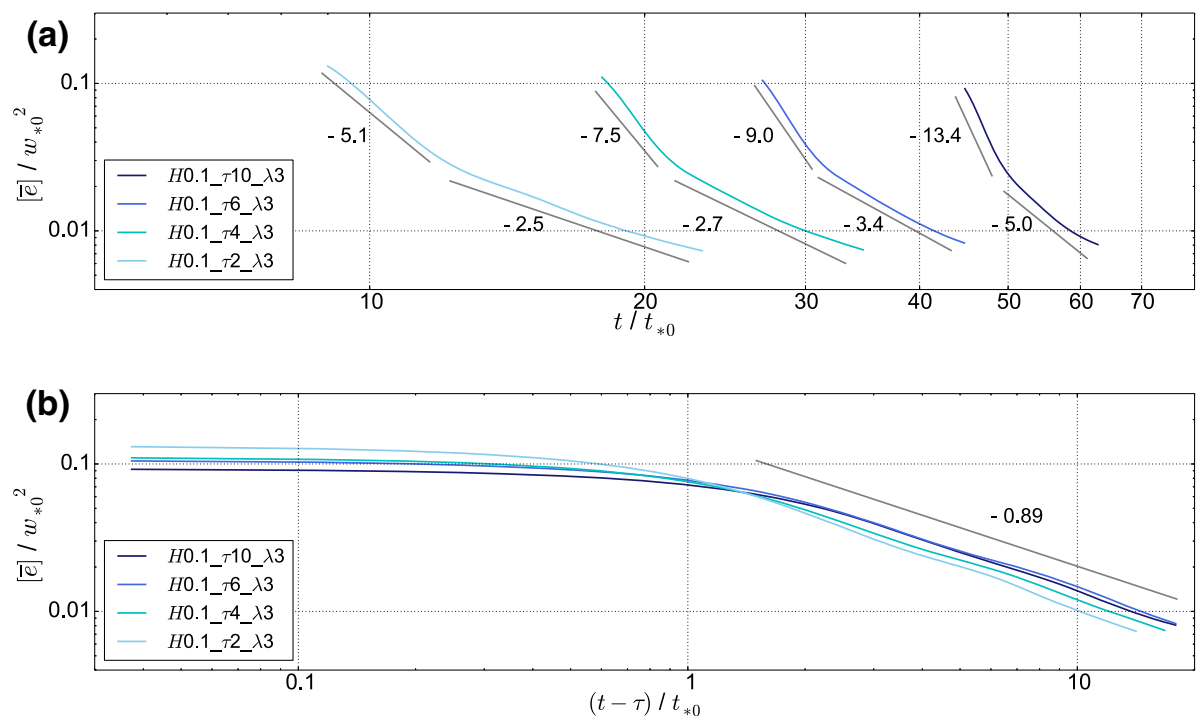

Fig. 8 Decay of TKE in runs $H 0.1 \_\tau 10 \_\lambda 3, H 0.1 \_\tau 6 \_\lambda 3, H 0.1 \_\tau 4 \_\lambda 3, H 0.1 \_\tau 2 \_\lambda 3$. a Traditional scaling framework. b Proposed scaling framework. For clarity, we plot only one line with a slope of -0.89 
Table 2 Decay exponents in the proposed scaling framework for all the runs

\begin{tabular}{lllll}
\hline$H\left(\mathrm{~K} \mathrm{~m} \mathrm{~s}^{-1}\right), \lambda\left(\mathrm{K} \mathrm{km}^{-1}\right)$ & $\tau=2 \mathrm{~h}$ & $\tau=4 \mathrm{~h}$ & $\tau=6 \mathrm{~h}$ & $\tau=10 \mathrm{~h}$ \\
\hline $0.1,3$ & 0.94 & 0.89 & 0.87 & 0.89 \\
$0.2,3$ & 0.98 & 0.98 & 0.87 & 0.86 \\
$0.3,3$ & 0.99 & 0.95 & 1.0 & 0.94 \\
$0.1,6$ & 0.96 & 0.93 & 0.89 & 0.72 \\
$0.2,6$ & 0.98 & 0.91 & 0.88 & 0.88 \\
$0.3,6$ & 0.92 & 0.85 & 0.92 & 0.87 \\
\hline
\end{tabular}

work. For each run, it results in two clearly distinguishable power-law regimes for the decay of TKE. These power laws and their exponents are presented in Fig. 8a. The power-law exponents increase with $\tau$. For the fast regime, the exponent varies from $\approx 5$ for $\tau=2 \mathrm{~h}$, to $\approx 13$ for $\tau=10 \mathrm{~h}$. For the slower decay regime, the corresponding exponent varies between 2 and 5. The decay rate is not similar in the traditional scaling framework. Results from the reanalysis of the data in the proposed scaling framework are shown in Fig. 8b. It is obvious that the proposed scaling establishes a single power-law exponent throughout the considered periods of time. The corresponding exponents (see Table 2) are in the order of 1 (and do not vary by more than \pm 0.04 ). The observed deviations in TKE magnitude between the $\tau 2$ and $\tau 10$ lines are much smaller than anything else presented in the traditional scaling as highlighted in Fig. 8a. More precisely, the lines in our scaling framework are almost parallel; that is not the case in the traditional scaling framework. The proposed transformation of time coordinate makes the decay rate largely independent of $\tau$.

In the previous analysis based on Fig. 8, we focused on the decay of TKE for a set of runs with the same convective initial condition. Now, we show the decay of TKE for five different convective initial conditions (Fig. 9). The resulting decay exponents from the proposed scaling framework are reported in Table 2. All these exponents are in the order of 1 , which is in line with our findings discussed above. The decay rate is therefore also independent of the convective initial conditions. One sees on the right panels of Fig. 9 that the curves are not perfect lines and show some fluctuations. However, the linear fit is a very good approximation for the first-order decay. Note that for the results presented in the traditional scaling framework in Fig. 9, we also find (not shown) a large dependence of the decay exponents on $\tau$, as in the analysis based on Fig. 8a.

\subsubsection{Sensitivity to the Normalization Factors}

As shown in the previous subsections, the decay exponent is independent of the initial convective condition, and $\tau$; the decay rate is therefore similar. However, the approximate initial time when similarity is valid depends on $\tau$. This is illustrated in Fig. 10a, where we normalize $[\bar{e}]$ by its initial value at the start of the evening transition (instead of $w_{* 0}^{2}$ ), but the time variable is still normalized by $t_{* 0}$. The initiation of the power-law regime is slightly delayed as $\tau$ increases. In order to take into account this dependence on $\tau$ in the time normalization, we define an eddy-turnover time at the start of the evening transition: $t_{\mathrm{ET}} \equiv\left(z_{i} /[\bar{e}]^{1 / 2}\right)_{\mathrm{ET}}$. As $H_{\mathrm{ET}} \equiv 0$, it is not possible to define the common convective eddy-turnover time at the start of the evening transition. The result is shown in Fig. 10b. The data for various $\tau$ are now collapsing to a single curve, and the power-law validity starts at $\approx 0.3 t_{\mathrm{ET}}$. Replacing $t_{* 0}$ with $t_{\text {ET }}$ does not change our results discussed in the previous sections (not shown). 
(a)

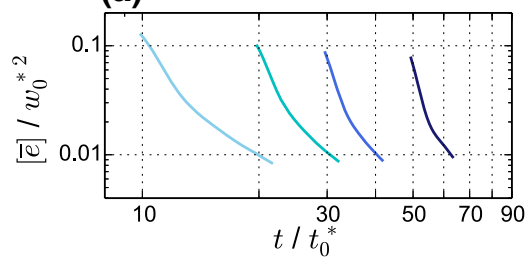

(c)

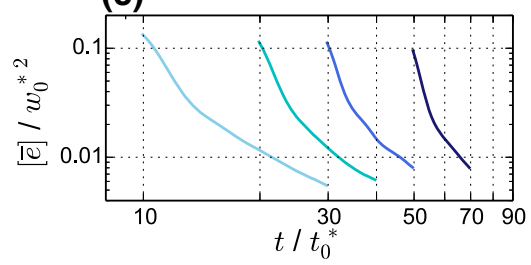

(e)

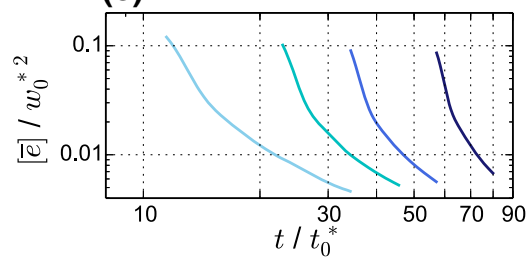

(g)

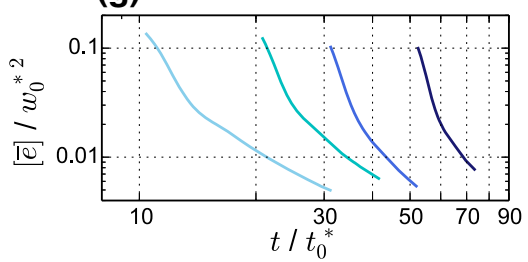

(i)

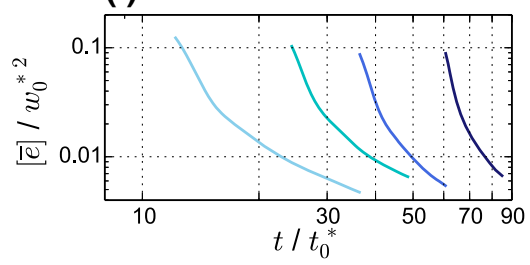

(b)

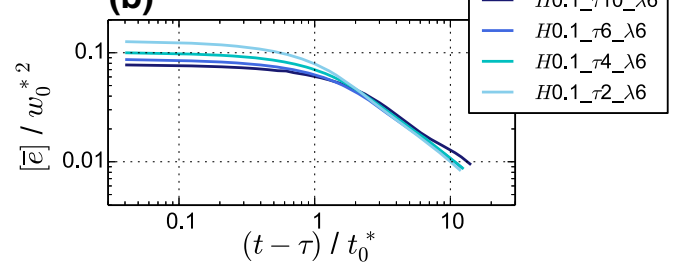

(d)
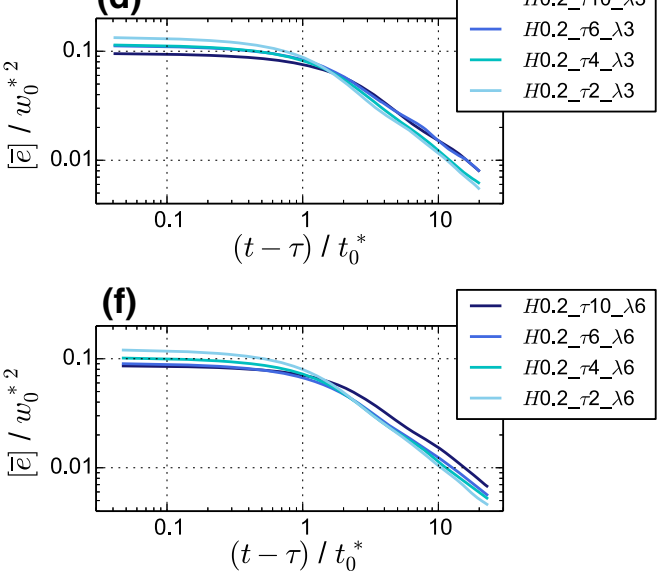

(h)
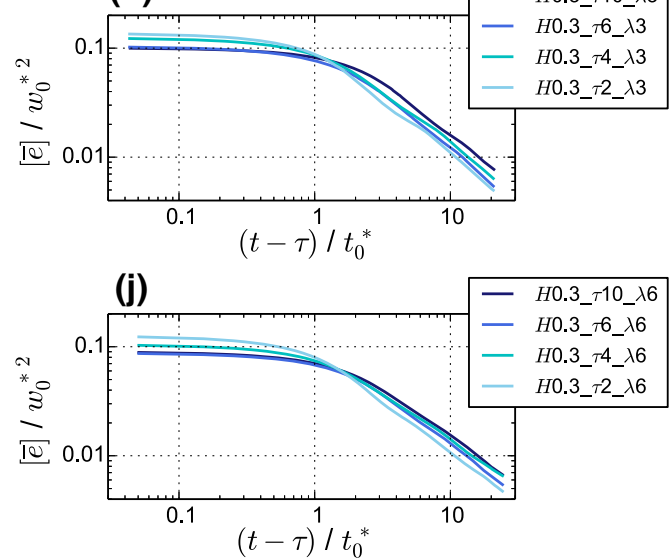

Fig. 9 Decay of TKE in the traditional and proposed scaling frameworks, left and right panels respectively. The pair of panels in each row is associated with a given convective initial condition. $\mathbf{a}$ and $\mathbf{b}$ Runs $H 0.2 \_\tau 10 \_\lambda 3, H 0.2 \_\tau 6 \_\lambda 3, H 0.2 \_\tau 4 \_\lambda 3, H 0.2 \_\tau 2 \_\lambda 3$. c and d Runs $H 0.3 \_\tau 10 \_\lambda 3, H 0.3 \_\tau 6 \_\lambda 3$, $H 0.3 \_\tau 4 \_\lambda 3, H 0.3 \_\tau 2 \_\lambda 3$. e and f Runs $H 0.1 \_\tau 10 \_\lambda 6, H 0.1 \_\tau 6 \_\lambda 6, H 0.1 \_\tau 4 \_\lambda 6, H 0.1 \_\tau 2 \_\lambda 6$. g and h Runs $H 0.2 \_\tau 10 \_\lambda 6, H 0.2 \_\tau 6 \_\lambda 6, H 0.2 \_\tau 4 \_\lambda 6, H 0.2 \_\tau 2 \_\lambda 6$. i and $\mathbf{j}$ Runs $H 0.3 \_\tau 10 \_\lambda 6, H 0.3 \_\tau 6 \_\lambda 6$, $H 0.3 \_\tau 4 \_\lambda 6, H 0.3 \_\tau 2 \_\lambda 6$

Note that while introducing the proposed scaling framework (paragraph 4 in Sect. 3.3), we chose to normalize time with the conventionally used $t_{* 0}$ and not with $t_{\mathrm{ET}}$, to emphasize the importance of properly defining the time origin. 

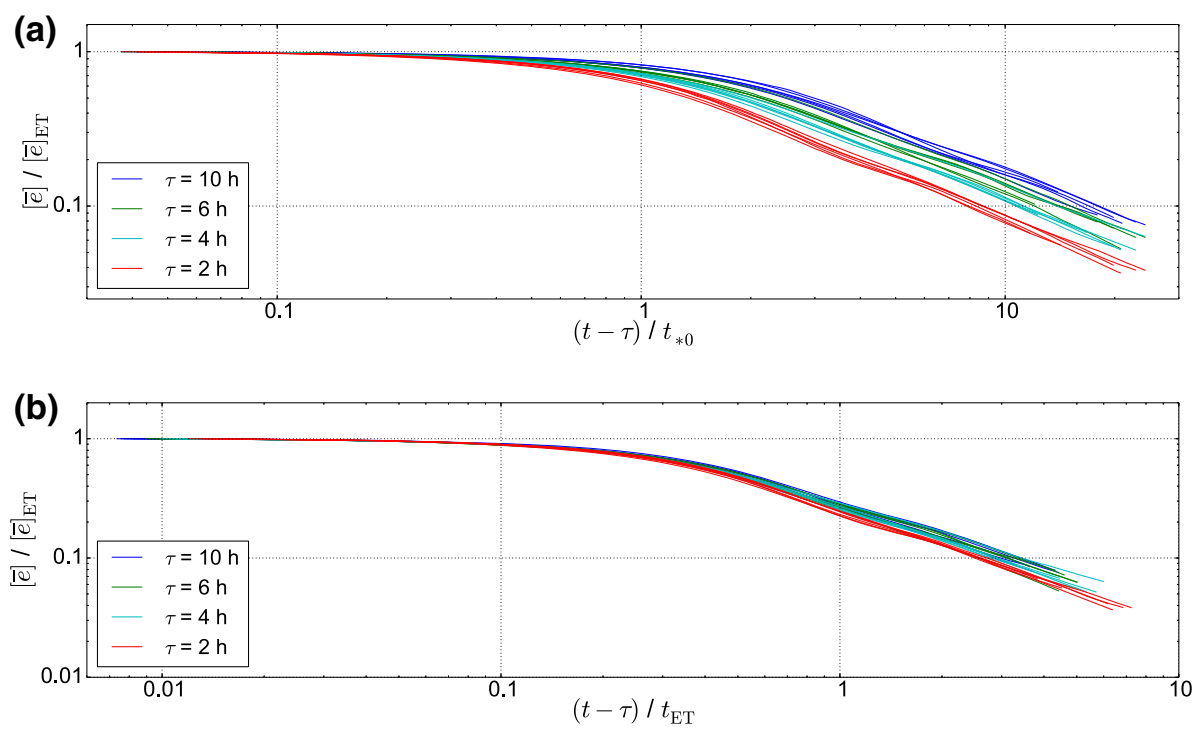

Fig. 10 Summarizing the decay of TKE for all the runs in the proposed scaling framework. Time and energy units for normalization are chosen as $t_{* 0}$ and $[\bar{e}]_{\mathrm{ET}}$ in $(\mathbf{a}) ; t_{\mathrm{ET}}$ and $[\bar{e}]_{\mathrm{ET}}$ in $(\mathbf{b})$

\section{Discussion}

\subsection{Simplifying Assumptions}

We discuss the first simplifying assumption, $H=0$. In reality, the heat flux becomes negative when the evening transition starts, and a SBL develops. The region of the atmosphere which is of interest is the layer above the growing near-surface stratification. We do not expect the first-order decay of the bulk TKE in this layer to depend on the presence or absence of a near-surface stratification. This is supported by the results shown in Fig. 11a, b, where one can see that the proposed scaling framework works also when we prescribe a negative heat flux. Note that the simulation with negative heat flux was run with 5-m isotropic grid spacing to take into account the influence of the stable surface layer. The available/limited computational resources did not allow us to conduct more simulations with negative heat flux.

The second simplifying assumption is zero geostrophic wind speed. In the TKE budget, the tendency changes according to the imbalance between buoyancy flux and dissipation. Increasing the shear generation will of course increase the TKE tendency. However, increasing TKE levels implies increasing dissipation. In addition, the response of the buoyancy flux to increasing shear is not evident. Using LES experiments and considering an abrupt decay of the heat flux, Pino et al. (2006) concluded: "In the case with no shear, turbulence decays faster." We conducted three additional runs with geostrophic wind speed $\left(u_{g}\right)$ of 2,6 , and $12 \mathrm{~m} \mathrm{~s}^{-1}$. Results are shown in Fig. 12 for one given convective initial condition, and three values of $\tau$ (2, 4 and $6 \mathrm{~h}$ ). Increasing the geostrophic wind speed reduces the decay rate, and a constant TKE level is reached toward the end of the simulation due to shear production for the high wind-speed cases. For the low wind-speed runs, $u_{g}=2 \mathrm{~m} \mathrm{~s}^{-1}$, the curves are parallel (as first approximation) to the curves for the runs without wind. Therefore, the suggested power-law scaling is still a good approximation for describing the decay. 

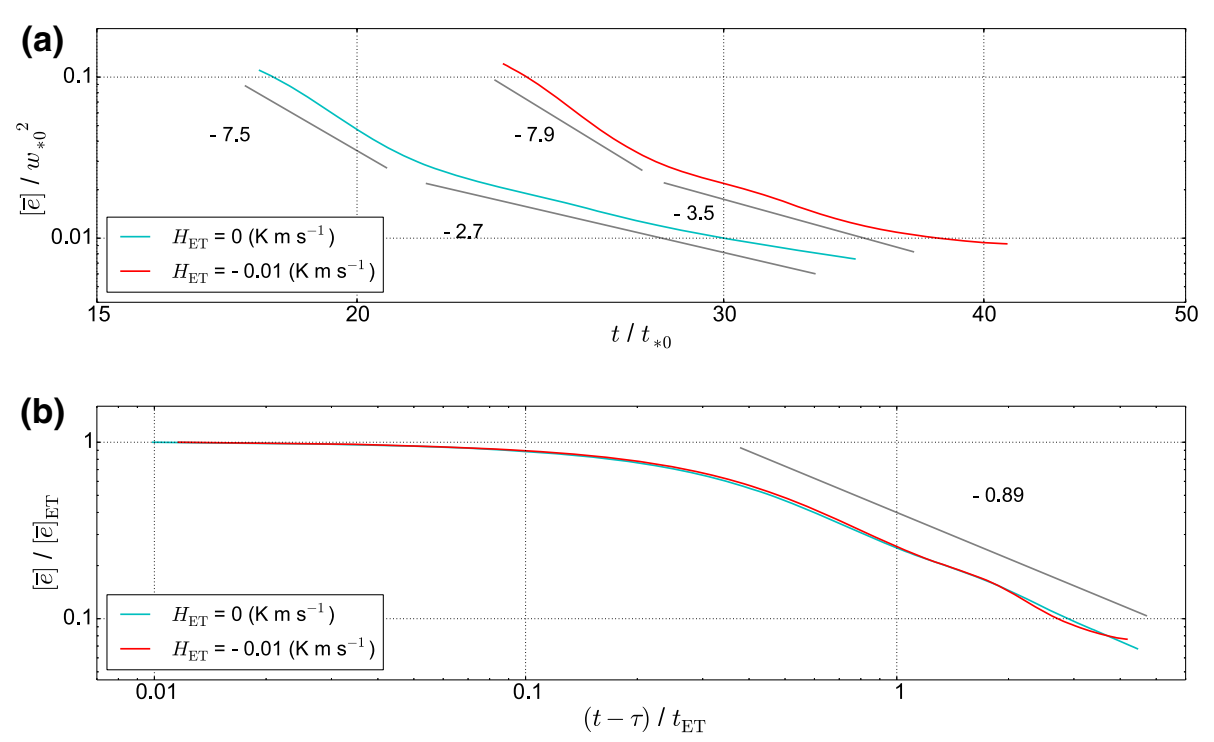

Fig. 11 Decay of TKE in run $H 0.1 \_\tau 4 \_\lambda 3$ with zero and negative $H$. a Traditional scaling framework. b Proposed scaling framework. In the case with negative $H$, TKE is averaged between the top of the surface stratification and the top inversion

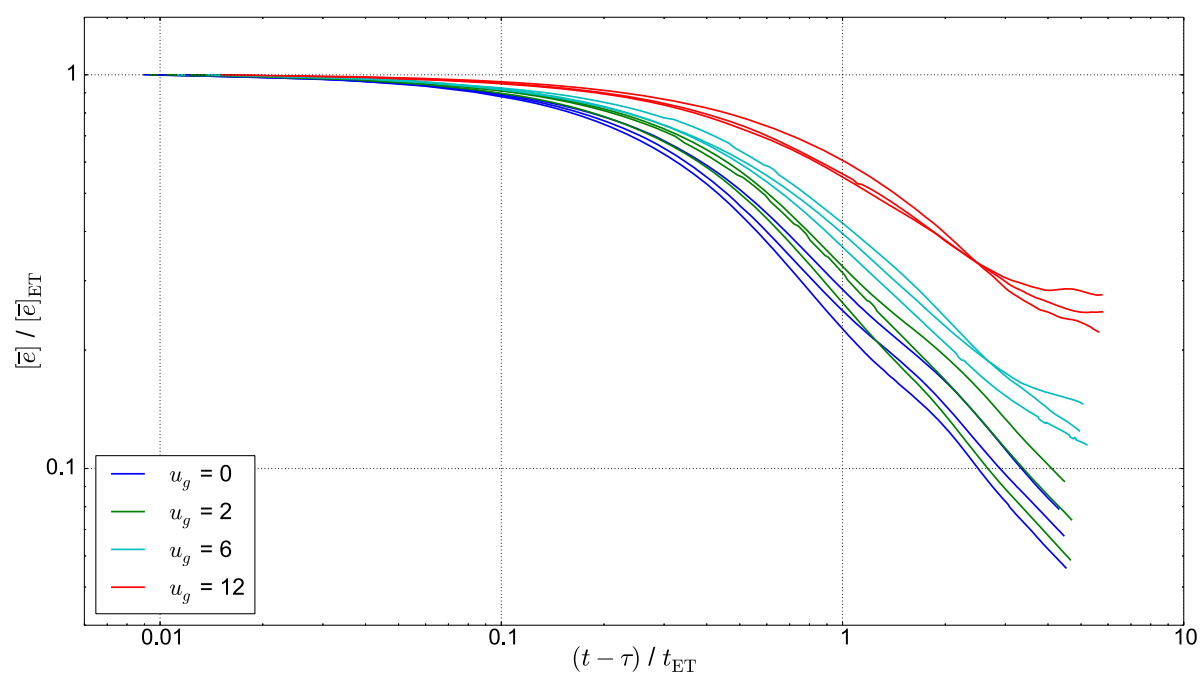

Fig. 12 Decay of TKE in the proposed scaling framework for runs $H 0.1 \_\tau 2 \_\lambda 3, H 0.1 \_\tau 4 \_\lambda 3$, and $H 0.1 \_\tau 6 \_\lambda 3$. Each colour is associated with a magnitude of the geostrophic wind. The colour code does not distinguish between $\tau=2,4$ and $6 \mathrm{~h}$

The power-law scaling suggested herein could be verified in the studies of Nadeau et al. (2011) and Rizza et al. (2013), by plotting TKE time series in a log-log plot with the time origin defined at the time when the heat flux changes sign. In laboratory or field experiments, mostly available pointwise measurements should be compared with care with our results 
which were deduced from the TKE average across the turbulent-layer depth. Knowledge of the height dependence of the decay rates would allow a fairer comparison with local measurements; a study currently underway addresses these issues. One would also question if the low magnitudes of TKE predicted from our LES are not too small to be measured. Taking run $H 0.1 \_\tau 4 \_\lambda 3$ with negative heat flux as an example and considering the time period when the power-law scaling applies, we found that the dimensional TKE is approximately in the interval $\left[0.02 \mathrm{~m}^{2} \mathrm{~s}^{-2}, 0.2 \mathrm{~m}^{2} \mathrm{~s}^{-2}\right]$. These values are within the capabilities of state-of-the-art measurements techniques.

\subsection{Sensitivity of the TKE Decay During the Evening Transition to the Abrupt/Gradual Heat-Flux Decay}

The proposed scaling framework is useful for comparing the decay of TKE when the heat flux is zero, between the cases where the previous heat-flux decay is abrupt or gradual. We conducted one experiment where the heat flux abruptly decays to zero, and found that a power law with an exponent of 1.2 is a good approximation for the decay of TKE, consistent with previous results (Nieuwstadt and Brost 1986; Sorbjan 1997). In the case where the heat flux gradually decays, previous results for the decay of TKE during the evening transition deduced from the traditional scaling framework, report decay exponents in the order of $6 \gg 1.2$ (Nadeau et al. 2011; Rizza et al. 2013). In these studies, where a heat loss to the surface was taken into account, strong damping of turbulence due to the growing SBL was suggested to justify the large decay rates of TKE. When presenting our results in the traditional scaling framework, and despite the absence of a stable stratification due to heat loss to the surface, we also find decay exponents much larger than 1.2. Therefore, damping of turbulence due to the growing SBL does not explain the large decay rates for the bulk TKE, deduced from the traditional scaling framework. In the proposed scaling framework, we find a unique power-law regime with an exponent in the order of 1 . When the heat flux is zero, we do not have a physical explanation for whether the decay of TKE should be faster or slower, depending on the abrupt or gradual previous decay of the heat flux. Yet, we proved that one approach to this problem is sensitive to the choice of the scaling framework.

\section{Conclusion}

We propose a new scaling framework for the decay of the bulk TKE above the near-surface stratification and below the capping inversion, in the core of the remnant daytime CBL, in low-wind situations,

$$
\frac{[\bar{e}]}{[\bar{e}]_{\mathrm{ET}}} \sim\left(\frac{t}{t_{\mathrm{ET}}}\right)^{-1} .
$$

where $[\bar{e}]$ is the TKE averaged over the boundary-layer depth $\left(z_{i}\right), t$ is the time coordinate, its origin is defined as the start of the evening transition, $[\bar{e}]_{\mathrm{ET}} \equiv[\bar{e}]$ at the evening-transition start, and $t_{\mathrm{ET}} \equiv\left(z_{i} /[\bar{e}]^{1 / 2}\right)$ at the evening-transition start.

We demonstrate in a concluding diagram (Fig. 13) that the decay of TKE from 0.3 of normalized time and onward is similar in our proposed scaling framework, whereas the traditional scaling framework does not reveal this similar behaviour. The traditional scaling framework should therefore no longer be considered suitable for describing the decay of TKE during the evening transition. During the afternoon transition, the decay of TKE scales with the time-evolving heat flux and inversion depth, up to $0.7 \tau$ approximately. Future work will 


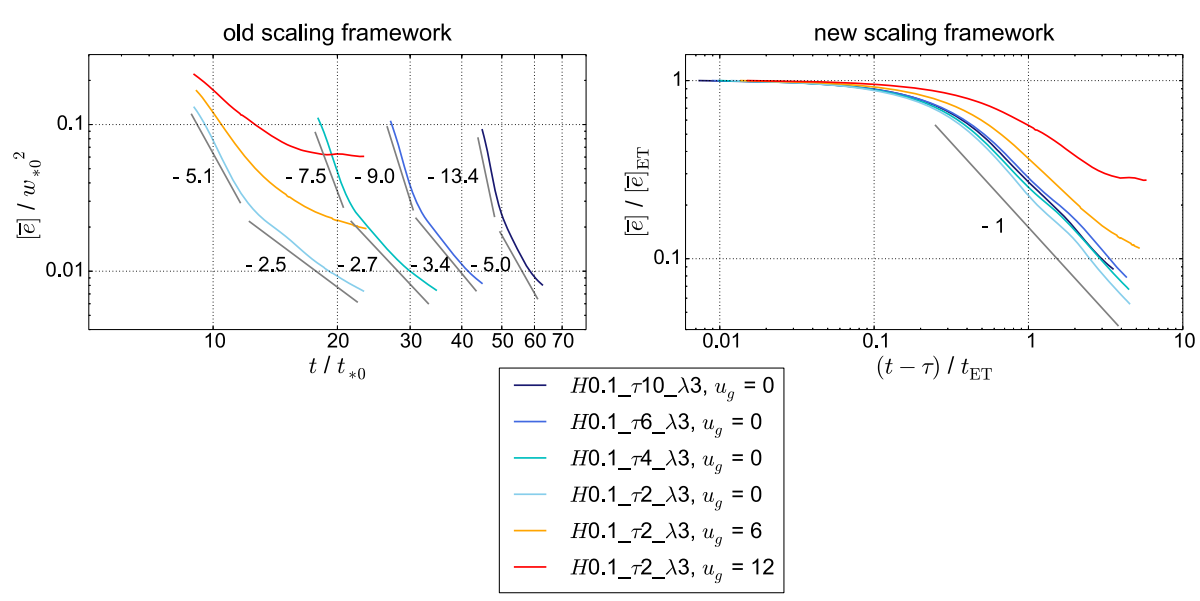

Fig. 13 Comparison of the two scaling frameworks. In the old scaling framework, the time origin is defined as the start of the afternoon transition. In the new scaling framework, we redefine the time origin as the start of the evening transition. $\tau$ is the duration of the afternoon transition

address the vertical dependence of the TKE decay, and the time change of energy distribution among different scales.

Acknowledgements We wish to thank the two anonymous reviewers for their helpful and constructive comments. CPU time was provided through the Norwegian Supercomputing Project NOTUR (II) Grant Nos. NN9528k and NN9506k.

Open Access This article is distributed under the terms of the Creative Commons Attribution 4.0 International License (http://creativecommons.org/licenses/by/4.0/), which permits unrestricted use, distribution, and reproduction in any medium, provided you give appropriate credit to the original author(s) and the source, provide a link to the Creative Commons license, and indicate if changes were made.

\section{References}

Batchelor GK, Townsend AA (1948) Decay of turbulence in the final period. Proc Roy Soc A 194:527-543

Couvreux F, Bazile E, Canut G, Seity Y, Lothon M, Lohou F, Guichard F, Nilsson E (2016) Boundary-layer turbulent processes and mesoscale variability represented by numerical weather prediction models during the BLLAST campaign. Atmos Chem Phys 16(17):8983-9002

Darbieu C, Lohou F, Lothon M, Vilà-Guerau De Arellano J, Couvreux F, Durand P, Pino D, Patton EG, Nilsson E, Blay-Carreras E, Gioli B (2015) Turbulence vertical structure of the boundary layer during the afternoon transition. Atmos Chem Phys 15:10,071-10,086

de Roode SR, Duynkerke PG, Jonker HJJ (2004) Large-eddy simulation: How large is large enough? J Atmos Sci 61:403-421

Deardorff JW (1970) Convective velocity and temperature scales for the unstable planetary boundary layer and for Rayleigh convection. J Atmos Sci 27:1211-1213

Deardorff JW (1980) Stratocumulus-capped mixed layers derived from a three-dimensional model. BoundaryLayer Meteorol 18:495-527

Djenidi L, Antonia RA (2015) A general self-preservation analysis for decaying homogeneous isotropic turbulence. J Fluid Mech 773:345-365

George WK (1992) The decay of homogeneous isotropic turbulence. Phys Fluids A 4(7):1492-1509

Goulart A, Degrazia G, Rizza U, Anfossi D (2003) A theoretical model for the study of convective turbulence decay and comparison with large-eddy simulation data. Boundary-Layer Meteorol 107:143-155

Huang MJ, Leonard A (1994) Power-law decay of homogeneous turbulence at low Reynolds numbers. Phys Fluids 6(11):3765-3775 
Ishida T, Gotoh T, Kaneda Y (2009) Study of high-Reynolds number isotropic turbulence by direct numerical simulation. Annu Rev Fluid Mech 41(3):165-180

Kanani-Sühring F, Raasch S (2015) Spatial variability of scalar concentrations and fluxes downstream of a clearing-to-forest transition: a large-eddy simulation study. Boundary-Layer Meteorol 155:1-27

Kolmogorov AN (1941) On degeneration of isotropic turbulence in an incompressible viscous liquid. Dokl Akad Nauk SSSR 31:538-540

Lavoie P, Djenidi L, Antonia RA (2007) Effects of initial conditions in decaying turbulence generated by passive grids. J Fluid Mech 585:395-420

Lothon M, Lohou F, Pino D, Couvreux F, Pardyjak ER, Reuder J, Vilà-Guerau de Arellano J, Durand P, Hartogensis O, Legain D, Augustin P, Gioli B, Lenschow DH, Faloona I, Yagüe C, Alexander DC, Angevine WM, Bargain E, Barrié J, Bazile E, Bezombes Y, Blay-Carreras E, van de Boer A, Boichard JL, Bourdon A, Butet A, Campistron B, de Coster O, Cuxart J, Dabas A, Darbieu C, Deboudt K, Delbarre H, Derrien S, Flament P, Fourmentin M, Garai A, Gibert F, Graf A, Groebner J, Guichard F, Jiménez MA, Jonassen M, Van den Kroonenberg A, Magliulo V, Martin S, Martinez D, Mastrorillo L, Moene AF, Molinos F, Moulin E, Pietersen HP, Piguet B, Pique E, Román-Cascón C, Rufin-Soler C, Saïd F, Sastre-Marugán M, Seity Y, Steeneveld GJ, Toscano P, Traullé O, Tzanos D, Wacker S, Wildmann N, Zaldei A (2014) The BLLAST field experiment: boundary-layer late afternoon and sunset turbulence. Atmos Chem Phys 14:10,931-10,960

Maronga B (2014) Monin-Obukhov similarity functions for the structure parameters of temperature and humidity in the unstable surface layer: results from high-resolution large-eddy simulations. J Atmos Sci 71:716-733

Maronga B, Gryschka M, Heinze R, Hoffmann F, Kanani-Sühring F, Keck M, Ketelsen K, Letzel MO, Sühring M, Raasch S (2015) The Parallelized Large-Eddy Simulation Model (PALM) version 4.0 for atmospheric and oceanic flows: model formulation, recent developments, and future perspectives. Geosci Model Dev $8: 2515-2551$

Mazzi B, Vassilicos JC (2004) Fractal generated turbulence. J Fluid Mech 502:65-87

Meldi M, Sagaut P (2013) Further insights into self-similarity and self-preservation in freely decaying isotropic turbulence. J Turbul 14:24-53

Mohamed MS, LaRue JC (1990) The decay power law in grid-generated turbulence. J Fluid Mech 219:195-214

Monin AS, Yaglom AM (1975) Statistical fluid mechanics, vol 2. The MIT Press, Cambridge

Nadeau DF, Pardyjak ER, Higgins CW, Fernando HJS, Parlange MB (2011) A simple model for the afternoon and early evening decay of convective turbulence over different land surfaces. Boundary-Layer Meteorol 141:301-324

Nieuwstadt FTM, Brost RA (1986) The decay of convective turbulence. J Atmos Sci 43:532-546

Nilsson E, Lothon M, Lohou F, Pardyjak E, Hartogensis O, Darbieu C (2016) Turbulence kinetic energy budget during the afternoon transition-Part 2: a simple TKE model. Atmos Chem Phys 16:8873-8898

Perot JB (2011) Determination of the decay exponent in mechanically stirred isotropic turbulence. AIP Adv 1:022104

Pino D, Jonker HJ, Vilà-Guerau de Arellano J, Dosio A (2006) Role of shear and the inversion strength during sunset turbulence over land: characteristic length scales. Boundary-Layer Meteorol 121:537-556

Raasch S, Franke T (2011) Structure and formation of dust devil-like vortices in the atmospheric boundary layer: a high-resolution numerical study. J Geophys Res Atmos 116:1-16

Rizza U, Miglietta MM, Degrazia GA, Acevedo OC, Marques Filho EP (2013) Sunset decay of the convective turbulence with large-eddy simulation under realistic conditions. Phys A Stat Mech Appl 392:4481-4490

Saffman PG (1967) Note on decay of homogeneous turbulence. Phys Fluids 10:1349

Schmidt H, Schumann U (1989) Coherent structure of the convective boundary layer derived from large-eddy simulations. J Fluid Mech 200:511-562

Sorbjan Z (1997) Decay of convective turbulence revisited. Boundary-Layer Meteorol 82:503-517

Sullivan PP, Patton EG (2011) The effect of mesh resolution on convective boundary layer statistics and structures generated by large-eddy simulation. J Atmos Sci 68:2395-2415

van Driel R, Jonker HJJ (2011) Convective boundary layers driven by non-stationary surface heat fluxes. J Atmos Sci 68:727-738

van Heerwaarden CC, Mellado JP (2016) Growth and decay of a convective boundary layer over a surface with a constant temperature. J Atmos Sci 73:2165-2177

von Karman T, Howarth L (1937) On the statistical theory of isotropic turbulence. Proc Roy Soc 164:192-215

Wicker LJ, Skamarock WC (2002) Time-splitting methods for elastic models using forward time schemes. Mon Weather Rev 130:2088-2097

Williamson JH (1980) Low-storage Runge-Kutta schemes. J Comput Phys 35:48-56 
Publisher's Note Springer Nature remains neutral with regard to jurisdictional claims in published maps and institutional affiliations. 